\title{
Zwei Tage Ärztekammersitzung für einen Neuanfang!
}

Die Ärztekammer - sozusagen das Parlament der FMH hat am 18. und 19. Mai ihre zweitägige Sitzung durchgeführt und ... der Neuanfang ist gelungen: Jetzt hat die FMH neue Statuten, und diese dürften grosse Auswirkungen auf die Beziehungen der FMH zu ihren Mitgliedern und auf ihre politische Präsenz haben. Das ist keineswegs nur Wunschdenken: Die Änderungen, die wir in unserem Verband vorgenommen haben, sind grundlegender Natur, und sie werden grosse Wirkung auf den Schwung haben, der uns antreibt.

Ich verspüre deshalb eine grosse Dankbarkeit gegenüber den Teilnehmerinnen und Teilnehmern dieser beiden Sitzungstage und gegenüber den zahlreichen Gruppierungen, die sie vertreten. Sie haben mit grosser politischer Reife auf die Zukunft gesetzt, auf eine FMH, die breiter abgestützt, anpassungs- und reaktionsfähiger und - weshalb eigentlich nicht? - auch phantasievoller ist.

Worum geht es? Hier die wichtigsten Neurungen:

- Da ist zuerst die Schaffung einer Delegiertenversammlung, bestehend aus 33 Mitgliedern, gestellt durch die Kantonalen Ärztegesellschaften, die medizinischen Fachgesellschaften sowie durch die verschiedenen «Bereiche» der Ärzteschaft (Ärztinnen und Ärzte in Weiterbildung, Chefärztinnen/Chefärzte, Ärztinnen usw.); die DV wird etwa alle zwei Monate das Gegenüber des Zentralvorstands sein, dessen strategische Zielsetzungen stützen, sie diskutieren und validieren, so dass sich dieser nicht mehr abgekapselt vorkommt, wie dies in der Vergangenheit allzu oft geschehen ist; hier geht es also um eine ganz grundlegende Neuerung, die die politische Präsenz der Ärzteschaft und die Qualität dieser Präsenz tiefgreifend verbessern wird.

- Und da ist das verstärkte politische Gewicht, das man dem Bereich «Bildung» verliehen hat. Seine Autonomie gegenüber dem Zentralvorstand wird verstärkt in seinen Aktivitäten, und in seinen Beschlussfassungen. Er wird vermehrt direkt der Ärztekammer gegenüber verantwortlich sein. Der Bereich «Bildung» ist zur Zeit ein politisch sehr sensibles Gebiet; es war uns deshalb sehr wichtig, seinem Verlangen nach verstärkter politischer Präsenz zu entsprechen.
Im übrigen wird eine Geschäftsprüfungskommission die bisherige Finanzkommission ablösen, die für die Rolle, die sie in einer Organisation von unserer Grösse hätte spielen sollen, nicht entsprechend ausgerichtet war. Weiter wurde eine Beschränkung der Amtsdauer für Zentralvorstandsmitglieder eingeführt, begleitet von einer klaren Unvereinbarkeit mit wirtschaftlichen Mandaten, und schliesslich wurden allenthalben die Formulierungen überarbeitet.

Von dieser Ärztekammer sind aber noch drei andere Punkte auf der Tagesordnung besonders erwähnenswert:

- Wir haben uns mit ein wenig Wehmut von Ursula Steiner verabschiedet, die aus dem Zentralvorstand zurücktritt; an ihrer Stelle wurde Ernst Gähler (Appenzell) gewählt - ein Porträt über ihn wird demnächst in der SÄZ erscheinen. Wir heissen ihn heute schon herzlich willkommen!

- Susi Stöhr wurde zur Vizepräsidentin gewählt - herzliche Gratulation!

- Das Budget 2007 wurde genehmigt - ohne Änderung der Grundbeiträge, aber mit einem sehr grossen Defizit. Wir werden dieses Defizit im 2007 auffangen können, aber es werden Überlegungen angestellt werden, welche die Budget- und Finanzpolitik der FMH bezüglich möglicher Ressourcen betreffen, und selbstverständlich auch bezüglich der steigenden Erfordernisse, die sich aus dem Aufgabenvolumen ergeben, das ständig grösser wird.

Wir haben also an dieser Ärztekammer Schritte von grösster Tragweite gemacht; wie nach einer guten Verhandlung war jeder und jede mit dem erreichten Ergebnis offensichtlich zufrieden - Beweis dafür ist die Einstimmigkeit (!) bei der Schlussabstimmung und der Applaus, der dieses Resultat unterstrich.

Es sieht ganz danach aus, als hätten wir bald keine Ausrede mehr, nicht die FMH zu sein, von der wir träumen!

Jacques de Haller, Präsident der FMH 